\title{
On velocity and temperature fluctuations generated by a turbulent grid and driven by buoyancy in open vertical channel
}

\author{
Malika Mahamdia $^{1, a}$, Michel Pavageau ${ }^{2}$ and Tahar Benabid ${ }^{1}$ \\ 1 Faculty of Physics, Theoretical and Applied Laboratory of Fluid Mechanics, University of Science and Technology \\ Houari Boumedienne, BP No. 32 El Alia 16111 Algiers, Algeria \\ 2 Institute Mining-Telecommunication, 37-39 rue Dareau, Paris, France
}

Received 8 December 2015, Accepted 20 September 2016

\begin{abstract}
This paper presents an experimental analysis of the statistical properties of velocity and temperature fluctuations generated downstream of two plan grids placed at the inlet of a large vertical open channel. A uniformly heated grid with a mesh spacing of $M_{\theta}=10 \mathrm{~cm}$ is placed underneath a second unheated turbulence-generating grid with a mesh spacing of $M=30 \mathrm{~cm}$. Buoyancy is the only driving force, and air is used as the working fluid and is heated from below The measurements of the generated turbulence are performed with the hot and cold-wire anemometry. It is shown experimentally that the turbulence behind the grid is inhomogeneous in its mean velocity and in its turbulence quantities. Even far downstream of the grid, the inhomogeneity of the mean velocity and the turbulence fluctuations persists. Regarding the longitudinal evolution of the mean turbulence, it is found that evolutions of the velocity and temperature variances are in contrast with the evolutions which are generally observed in forced convection turbulence grids. However, in the first part of the flow, $x / M \leq 13.33$, the mean temperature variance decreases and evolves in agreement with the variance generally observed in homogeneous and isotropic grid turbulence flow.
\end{abstract}

Key words: Experiment / free convection / grid turbulence / open vertical channel

\section{Introduction}

The ability of turbulence to mix and transport one or more scalars within a fluid is particularly relevant to a variety of engineering applications including combustion, pollution dispersion and environmental problems. One of the aims of turbulence research is to predict the structure and transport characteristics of a scalar in a turbulent flow field, which could be temperature, humidity, or any chemical species. Understanding the behaviour of thermal fluctuations for example is a necessary first step in understanding the processes of transport or turbulent heat flux mixing. However, the fluid mechanics that governs turbulent mixing involves other phenomena for which the details are not yet fully understood. Indeed, for more specific subjects that require an understanding of how the scalar quantities can evolve in the presence of dynamically

${ }^{a}$ Corresponding author: mmahamdia2005@yahoo.fr active buoyancy forces, our comprehension and ability to predict such turbulent mixing are limited.

One could assume that the extension of our knowledge of the velocity field to which a scalar is added could be achieved without difficulty. However, both theoretically and experimentally this problem has proved to be difficult to study, even for the simplest types of flow.

The simplest case for studying a transport scalar in a turbulent flow is that of decaying scalar in approximately isotropic grid-generated turbulence. Grid-generated turbulent flows have been the subject of numerous turbulence investigations due to their statistical properties, the translational invariance of homogeneous turbulence, and decay processes. This type of turbulent flow has constituted the ideal framework for understanding and modelling turbulent flow phenomena. However the usual models of closure of turbulent equations come mainly from experiments conducted in mixed or forced convection. For turbulent free flow of natural convection, the 
question of the validity of the physical analysis that is made through these equations remains posed.

In forced convection, generally a thermal fluctuation occurs by imposing a mean temperature gradient to the turbulence generating grid, by using an array of finely heated wires placed downstream of the turbulence generating grid or by heating the grid itself. The decaying grid turbulence, in homogeneous and isotropic flow and with or without heating continues to be the basic reference for the development of many theories and turbulence models The earlier works of Taylor [1], Batchelor [2], Corrsin [3] and his co-workers, Mills et al. [4,5], Warhaft and Lumley [6] and Sirivat and Warhaft [7] which pioneered the development of turbulence theory were revisited by a number of authors, e.g., George [8], Gibson and Dakos [9], and more recently in studies conducted by Lavoie et al. [10], Antonia et al. [11], Lee et al. [12]. The dependency on initial conditions such as Reynolds number, mesh size, solidity, grid rod shape and surface roughness are clearly identified. Objective methods have been proposed by authors cited above to reduce significantly this dependency. In forced convection, the values of thermal and kinetic energies $\left\langle q^{2}\right\rangle$ and $\left\langle\theta^{2}\right\rangle$ are readily determined from measurements in uniformly heated grid turbulence, as reported by Warhaft-and Lumley[6].However, their decay vary enormously from experiment to experiment, either with the power input to the heating device, or, with the initial conditions. These problems might be avoided by the generation of a self-sustaining temperature field, sufficiently far from the heat source for the development of $\left\langle\theta^{2}\right\rangle$ to be independent from the initial conditions. To obtain the best isotropic and uniform upstream flow, many of these studies conducted by ComteBellot and Corrsin [13] have used a wind tunnel with contracted walls. Since first works mentioned above, until recent progress in modelling scalar fluctuations, (e.g., Viswanathan and Pope [14], the scalar transport has been treated as a "passive" scalar, having no dynamic effect on the flow. The experiments to date have dealt exclusively with small temperature gradients for which buoyancy forces are negligible.

The experimental facilities of our present study allowed us examine a natural convection flow in a laboratory environment, a mixing of a turbulent active scalar and finally the possibility of reaching high Reynolds numbers. There is no fan, so the air is purely driven by thermal convection. This is the simplest situation in which we can isolate and study the effects of buoyancy on the transport of turbulent energy. It may be observed that although several studies have been conducted in the wind tunnel, to our knowledge no studies have heretofore been investigated in turbulent free convection. One of our first aims is to provide-a-statistic-description-of-grid-flow-inducedby buoyancy, -and to-understand the behaviour of basic characteristics such as the thermal and dynamic turbulent energies. All of the information gained from this work is presented and discussed in the following sections. Eventually, even though measurement techniques and numerical methods have been significantly improved in the last decade, the experimental investigations in turbulent natural convection remain a challenge. The reason is that, low velocities and temperature are difficult to measure, and ideal homogeneous and isotropic flow conditions are challenging to achieve.

\section{Outline of the experimental facility and measurement techniques}

\subsection{Experimental facility}

The experiments are performed in a vertical open channel with a total height $\mathrm{H}$ of $13 \mathrm{~m}$, and a large test section with dimensions DxD of $3 \mathrm{~m} \times 3 \mathrm{~m}$, as described in detail in Figures 1a and 1b. Measurements are performed in the Fluid Mechanics Laboratory (University of Nantes-Ecole Centrale of Nantes, France). The test channel is divided into two parts situated on either side of the plan grids. The first part, which is $3 \mathrm{~m}$ high, is located in the basement of the building and is used to produce fresh air. To reduce the vortices and lateral mean velocity fluctuations, the air then passes through a set of honeycombs. The second part is the test portion, which extends from the grids to the channel exit. The lateral walls consist of two metal sheets $2 \mathrm{~mm}$ thick and $10 \mathrm{~cm}$ apart; the gap is filled with glass wool to limit the heat transfer between the inside and the outside channel. A thermal biplane grid of a square mesh with $M_{\theta}=10 \mathrm{~cm}$ and a coefficient solidity of $28 \%$ is used to electrically heat the flow. The mesh consists of a perpendicular arrangement of two sets of 29 resistive sheathed rods that are $15 \mathrm{~mm}$ in diameter. The electrical circuit is manually controlled to maintain a constant power input and to create a constant mean temperature rise across the grids. Two thermometers located 15 mesh lengths upstream and downstream from the grids measure approximately $\Delta T=10 \mathrm{~K}$ for $80 \mathrm{KW}$ of input power; the thermal grid surface reaches $300{ }^{\circ} \mathrm{C}$. The biplane grid-generated turbulence, with a square mesh of $M=30 \mathrm{~cm}$ and a solidity coefficient of $34 \%$ is placed at 1.5 mesh lengths from the heated grid; its role is to create and pilot the turbulence. This experimental setup creates a free turbulence field flow at a reasonably high Reynolds number, $R e_{M}=\langle U\rangle M / \nu$, where $\langle U\rangle$ is the mean flow velocity in the test section and $\nu$ is the kinematic viscosity. To measure the mean temperatures inside and outside the channel, 15 type chromel-alumel calibrated thermocouples are vertically introduced using thin cables. To reduce possible effects of the channel exit, the measurements reported here stop at approximately one channel width from the outlet.

\subsection{Measurement techniques}

The instantaneous temperature measurements within the flow are made using Wollaston-process platinum wires that are $1 \mu \mathrm{m}$ in diameter and $2 \mathrm{~mm}$ long. These cold wires operate with a constant current without thermal 


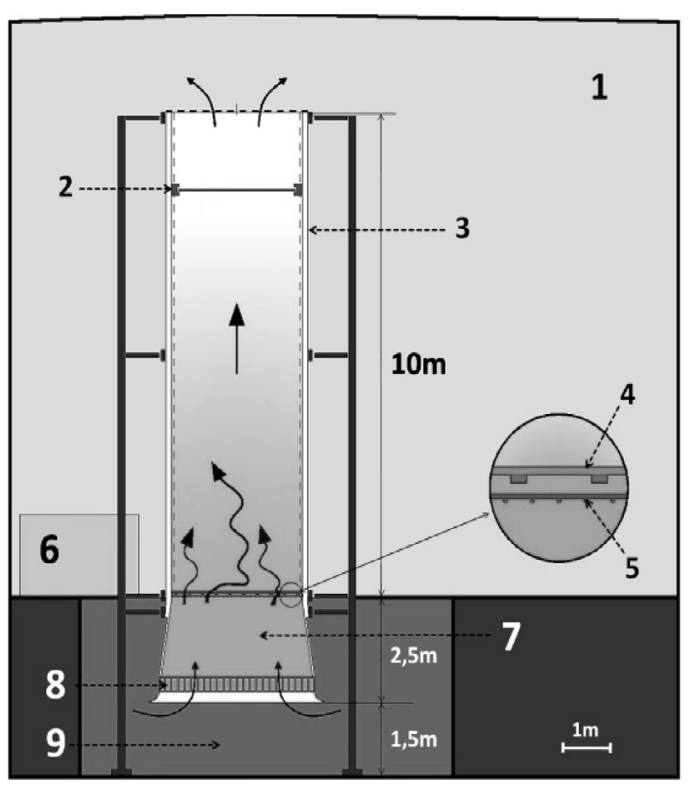

(a)

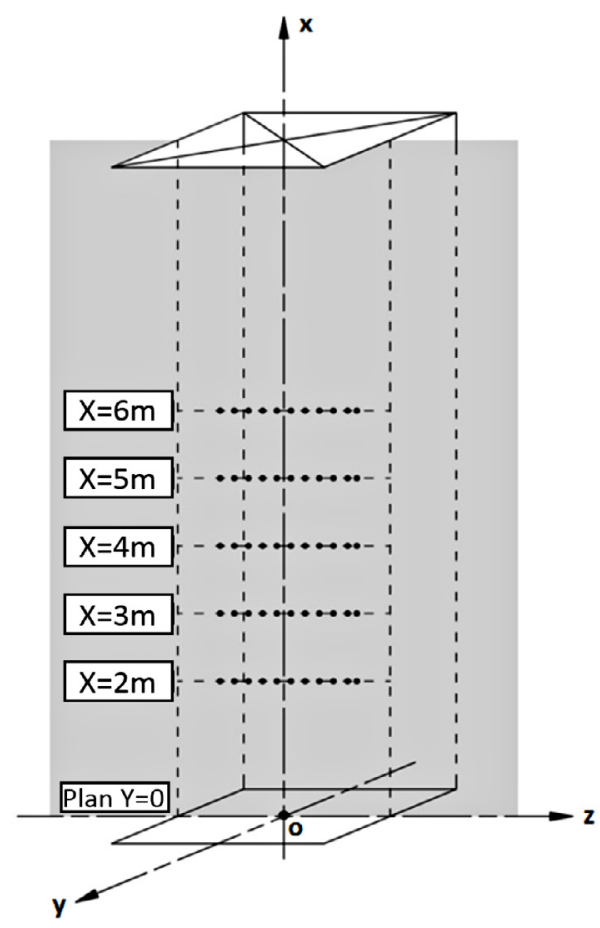

(b)

Fig. 1. (a) Cross section in the xoz plane showing the different parts of the experimental apparatus. (1) experiment hall, (2) carriage moving probes, (3) insulated walls, (4) dynamic grid, (5) thermal grid, (6) heating control system, (7) input channel, (8) filter honeycomb and (9) basement of the building. (b) A sketch of the measuring points positions. Along $z / M=2 ; 2.66$; $3.33 ; 4 ; 4.66 ; 5.33 ; 6 ; 6.66 ; 7.33 ; 8$ and 8.43 .

compensation for thermal inertia; the current goes as low as $140 \mu \mathrm{A}$ to ensure that the velocity sensitivity of the cold wires is negligible. The cold-wire time constant and cut-off frequency $(-3 \mathrm{~dB})$ are estimated to be $6.3 \times 10^{-5} \mathrm{~s}$ and $2.500 \mathrm{KHz}$, respectively. The length-to-diameter ratio is 2.000 so that the conduction heat losses through the prongs and the supports have no significant influence on the response of the sensors.

Velocity fluctuations were measured with tungsten hot wires $5 \mu \mathrm{m}$ in diameter and $3 \mathrm{~mm}$ long. The sensors were operating using non-linearized DISA 56C17 constant-resistance bridges at an overheat ratio of 0.6 to reduce the temperature sensitivity of the sensors. The wire time constants were on the order of $5 \times 10^{-4} \mathrm{~s}$, with a length-to-diameter aspect ratio of 600 to minimize the spurious nonlinearity due to the end effects. The lateral velocity components, $\mathrm{W}$, measured with a $90^{\circ} \mathrm{X}-$ wire probe. Therefore, the flow presented here is vertical and unidirectional in the mean. The outputs signals from the anemometers and the thermometers were low-pass filtered with a cut-off frequency to remove high-frequency electrical noise. The signals were then digitally recorded on a computer using the ACODAS data acquisition software from Data Instruments Inc. The calibration laws were then applied for data conversion into physical units. Given the full-scale voltages used in the experiments, the 12-bit A/D converter provided resolutions equivalent to $5 \times 10^{-5} \mathrm{~m} . \mathrm{s}^{-1}$ and $10^{-3} \mathrm{~K}$ for velocity and temperature, respectively. The data were acquired at a sampling frequency of $200 \mathrm{~Hz}$ over $120 \mathrm{~s}$. In practice, the instantaneous temperatures are determined first because they intervene in the calculation of the velocity. Because of the low turbulence intensity $\left(\left\langle u^{2}\right\rangle^{1 / 2} /\langle U\rangle<3 \%\right)$, the use of Taylor's hypothesis to convert temporal to spatial derivatives in the $x$ direction should be satisfactory.

Approximately, $3 \mathrm{~h}$ of heating were needed in order for the ambient fluid either at rest, and the mean flow, with a slightly temporal variation rate. The thermocouple measurements indicated that, the mean vertical gradient of the ambient mean temperature was estimated to be about $1 \mathrm{~K} . \mathrm{m}^{-1}$, it corresponds to a permanent stratification. The mean vertical gradient of temperature was nearly constant within the investigated area it is equal to $-0.3 \mathrm{~K} . \mathrm{m}^{-1}$ thus representative of an unstable thermal stratification.

\section{Results and discussion of the mean and fluctuating quantities}

The results refer to a Cartesian coordinate system located downstream of the grid generated turbulence; the upper surface of the turbulence grid coincides with the Ozy plan. The origin is at the left wall of the channel, $x$ is the vertical axis (positive upwards) and $z$ is the horizontal axis (positive to the right side). The transverse variations 


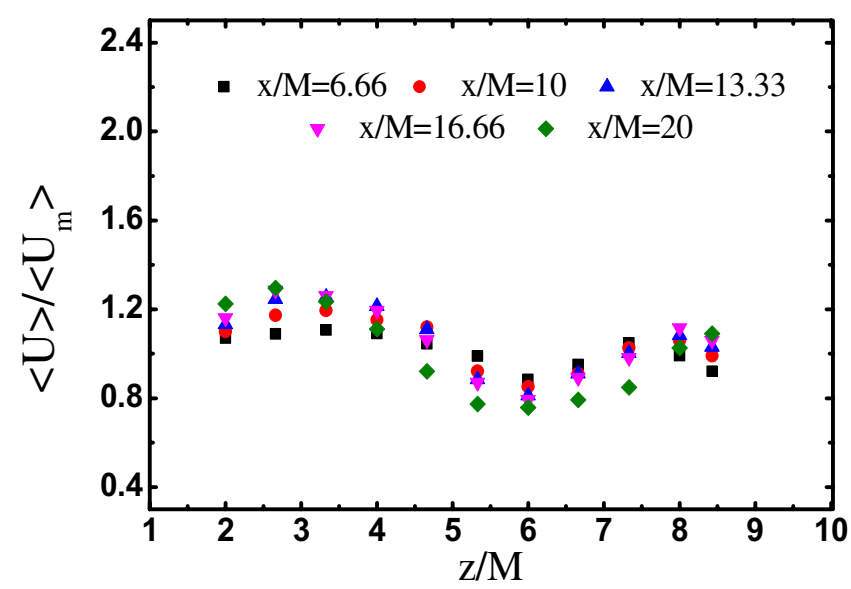

Fig. 2. Transversal profiles of the mean vertical velocity component at five positions downstream the grid $\left\langle U_{m}\right\rangle$ the centreline mean velocity component $(z / M=6)$.

of velocity, temperature and their fluctuations are plotted for five test-stations as shown in Figure 1a, using 11 measuring points included in the interval $2<z / M<8.43$, located away from the boundary layer walls. The temperatures and velocities are normalized by the mean values $\left\langle T_{m}\right\rangle$ and $\left\langle U_{m}\right\rangle .\left\langle T_{m}\right\rangle$ is the centre-line mean temperature and $U_{m}$ is the centre-line mean velocity component. In the following, we present the main characteristics of the mean velocity and temperature fields as well as their fluctuations.

\subsection{The mean velocity and temperature fields}

Normally, the systematic checks on the homogeneity of the flow are carried out before undertaking any statistical analysis for which homogeneity is required. However, the literature shows that although grid-generated turbulence was utilized in many experimental turbulence investigations, its ihomogeneity did not attract enough. Sirivat and Warhaft [7], in studying vertical wind tunnel flow generated by a turbulence grid, noted that good transverse homogeneity is never attained. In our experiment, the profiles of the mean streamwise velocity components depicted in Figure 2 show an asymmetry and inhomogeneity in the transverse distribution of the mean vertical velocity field. This asymmetry comes mainly from the initial conditions of the generation of the flow.

The transversal velocity gradients that appear on both sides of the profile of Figure 2 clearly lead to the acceleration of the flow in the areas near the channel walls. The minimum of velocity observed in the vicinity of the median plane indicates the existence of a weak circulation region of the fluid. The same comments were reported by Ayinde et al. [15]. Uberoi and Wallis [16] shown that the inhomogeneity profile is conserved and propagated in the streamwise flow. Ertunc et al. [17] recently noted that the inhomogeneity of the mean velocity in experiments and simulations with uniform grid structures had a tendency to decrease far away from the grid. Also, it is

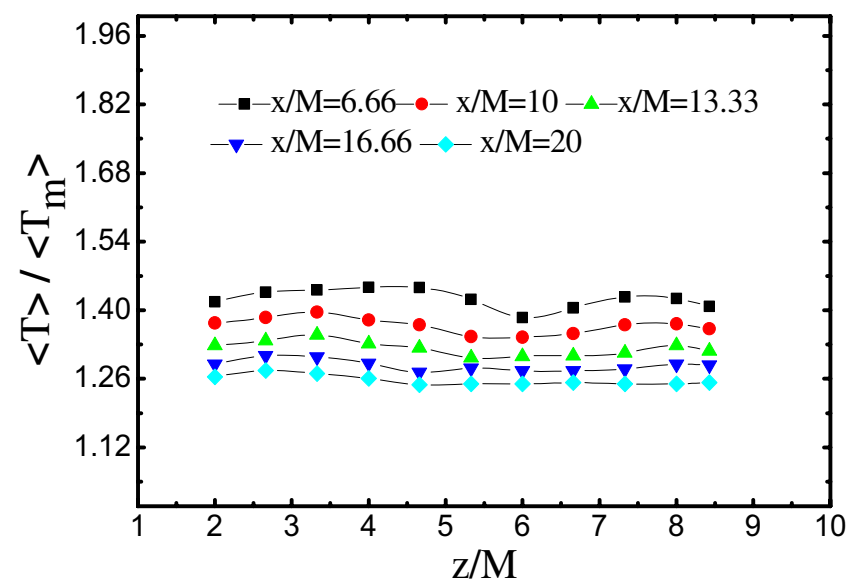

Fig. 3. Transversal mean temperature. $\left\langle T_{m}\right\rangle$ the mean centreline mean temperature.

important to remember that in order to ensure the homogeneity of the turbulent forced flow field in the wake of a grid, Corrsin [18] suggested that the larger the $D / M$ ratio is, the smaller the effect of the walls on the measured data is expected to be. Moreover, the measurements should be taken far downstream because the turbulence becomes homogeneous after at least 40 mesh sizes downstream of the grid. In our experimental facility given that the height of our test section could not exceed 10 meters with mesh spacing of $M=30 \mathrm{~cm}$, these conditions are difficult to achieve. For this purpose, the present data must not be compared with those deduced for homogeneous turbulence.

Figure 3 shows that the appearance of transversal mean temperature profiles at different stations is similar regardless of the altitude. However, a slight inhomogeneity is observed in the transverse distribution of the mean temperature up nearby $x / M=13.33$. This configuration of the mean temperature field comes mainly from the initial conditions of the generation of the flow. Various attempts tested to improve this inhomogeneity have been ineffective. Nevertheless, in contrast to what is observed for velocity fields, the thermal field inhomogeneity decreases with altitude. This streamwise homogenization of the thermal field was also observed in the recent DNS study of Djenidi and Tardu [19], which showed that while the lateral inhomogeneity was high in the region close to the grid $x / M<10$, it decreased with increasing distance from the grid. Figure 4 shows that the mean axial temperature decreases linearly downstream of the grid along the channel. $\left\langle T_{z}\right\rangle$ is the average temperature in horizontal planes following $z$ direction.

\subsection{Distributions of fluctuations and mean velocity variances}

Figures $6 \mathrm{a}$ and $6 \mathrm{~b}$ show the transverse profiles of the turbulence intensities, $I_{u}=c\left\langle u^{2}\right\rangle^{1 / 2} /\langle U\rangle$ and $I_{w}=$ $\left.\left\langle w^{2}\right\rangle^{1 / 2} /\langle U\rangle\right)$. In the vicinity of the central axis, regardless of the distance downstream of the grid, a maximum 


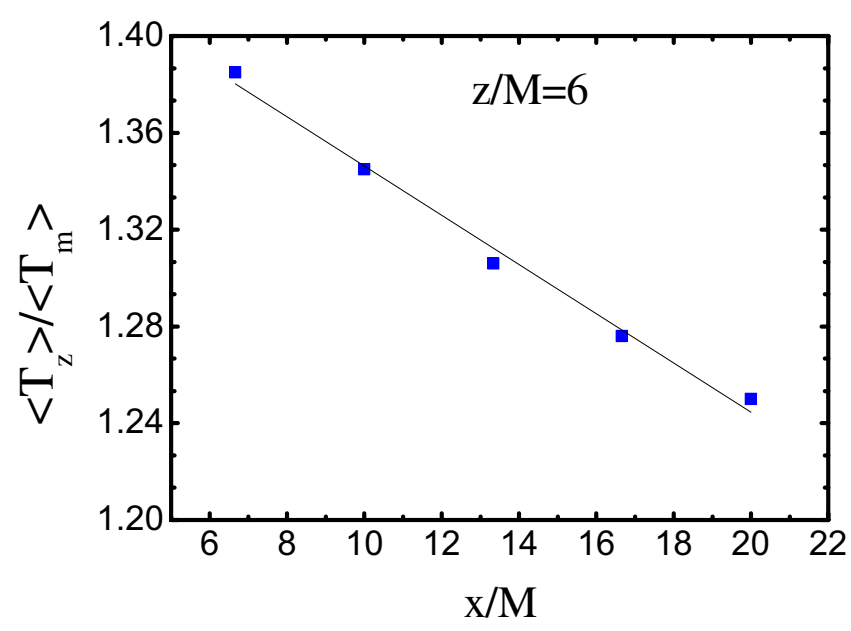

Fig. 4. Evolution of the mean temperature variance in the neighbourhood of the centre-line of the channel $(z / M=6)$, $\left\langle T_{m}\right\rangle$ the centre-line mean temperature.

is observed that corresponds to the minimum mean velocity. The reason is that, the energy exchange in the flow involves a loss to the mean flow and an increase to the turbulent field. At $x / M=6.66$, the standard deviation from the mean is $\pm 2 \%$, which contrasts with $\pm 5.5 \%$ at $x / M=20$. The simplest assessment of largescale anisotropy is achieved by comparing the ratio of the streamwise and transverse r.m.s. velocity components, sometimes referred to as an isotropy factor. In spite of the data scattering shown in Figure 7a, the mean value of the isotropy factor is close to 1.15 (green line), which is similar to the known value of 1.2 found in the classic case of grid-generated turbulence for a non-contracted channel. Mohamed and LaRue [20], with a contracted channel, noted that $I_{u} / I_{w} \approx 1.11$.

The anisotropy factor plotted in Figure $7 \mathrm{~b}$ increases far downstream of the grid, confirming the role played by the buoyancy in the anisotropy of turbulent flow. Through these figures, we can consider that the flow studied here highlights the anisotropic nature of turbulent free convection flows.

\subsubsection{Kinetic energy transport equation of the normal component (budget $\left\langle u^{2}\right\rangle$ )}

Figure 5 described the evolution of the vertical mean turbulent kinetic energy, in way to express a local situation that could be obtained if the flow was homogeneous in each horizontal plan; each value of $\left\langle u^{2}\right\rangle$ is equivalent to the integral of the values over a transversal measurement line. We notice that, in the immediate vicinity of the grid, the turbulence decays, which is somewhat consistent with what is generally observed in grid turbulence, but it increases beyond this distance.

We evaluate the terms of the kinetic energy turbulence in the transport equation of $\left\langle u^{2}\right\rangle$ to provide an explanation for this persistence increase far downstream of the grid. For each budget, the different terms were expressed

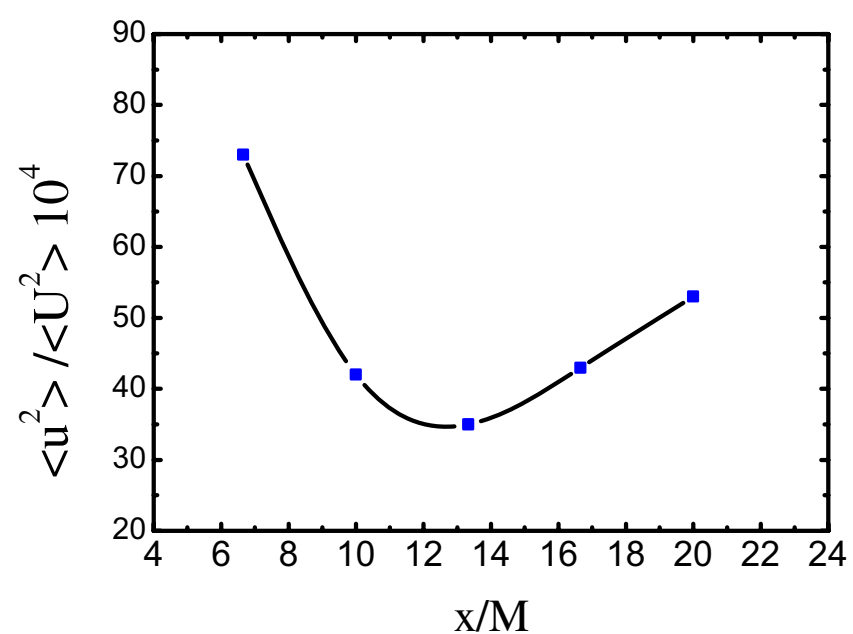

Fig. 5. Evolution of the streamwise mean velocity variance at $z / M=6$ neighbourhood of the centre-line of the channel.

in a simplified form resulting from the flow transversal homogeneity hypothesis. For a stationary, incompressible flow, the turbulent kinetic energy transport equation is written in budget form, facilitating the physical phenomena interpretation associated with each term (Craft et al. [21]).

$$
\frac{D\left\langle u_{i} u_{i}\right\rangle}{D t}=G_{i j}+d_{i j}+\varphi_{i j}+\epsilon_{i j}
$$

The left-hand side contains the temporal variation and the convection by mean velocity terms. For stationary turbulence, the left-hand side is reduced to transport by the mean flow term. The right-hand side is broken down into different terms with distinct physical meanings. The symbols, $P_{i j}, G_{i j}, d_{i j}, \phi_{i j}$ and $\varepsilon_{i j}$ denote the production terms, the buoyant production terms, the diffusion terms, the correlation pressure-deformation terms and the dissipation rate. The final dimensional of the normal component $\left\langle u^{2}\right\rangle$ budget is given by

$$
\begin{gathered}
\underbrace{\langle U\rangle \frac{\partial\left\langle u^{2}\right\rangle}{\partial x}}_{1} \approx \underbrace{-2 \frac{\partial}{\partial x}\left\langle\frac{p u}{\rho}\right\rangle}_{2}-\underbrace{\frac{\partial}{\partial x}\left\langle u^{3}\right\rangle}_{3}+\underbrace{2 \nu \frac{\partial}{\partial x}\left(\frac{\partial\left\langle u^{2}\right\rangle}{\partial x}\right)}_{D_{u u}} \\
-\underbrace{2\left\langle u^{2}\right\rangle \frac{\partial\langle U\rangle}{\partial x}}_{5}+\underbrace{2\langle u \theta\rangle \frac{g}{\left\langle T_{m}\right\rangle}}_{\Phi_{u u}}+\underbrace{2\left\langle\frac{p}{\rho} \frac{\partial u}{\partial x}\right\rangle}_{7}-\underbrace{\frac{2}{3} \epsilon_{u}}_{8}
\end{gathered}
$$

where $\langle U\rangle$ is the mean velocity in the streamwise direction, $g$ is the acceleration due to gravity, $\left\langle T_{m}\right\rangle$ is the ambient temperature (reference temperature), $u$ is the longitudinal velocity fluctuation component, $\theta$ is the temperature fluctuation, $p$ is the fluctuating pressure, $\epsilon_{u}=\nu\left\langle(\partial u / \partial x)^{2}\right\rangle$ is the dissipation rate of the velocity fluctuations and $\nu$ is the kinematic viscosity. The rate change of $\left\langle u^{2}\right\rangle$ is thus due to the three diffusion terms of 

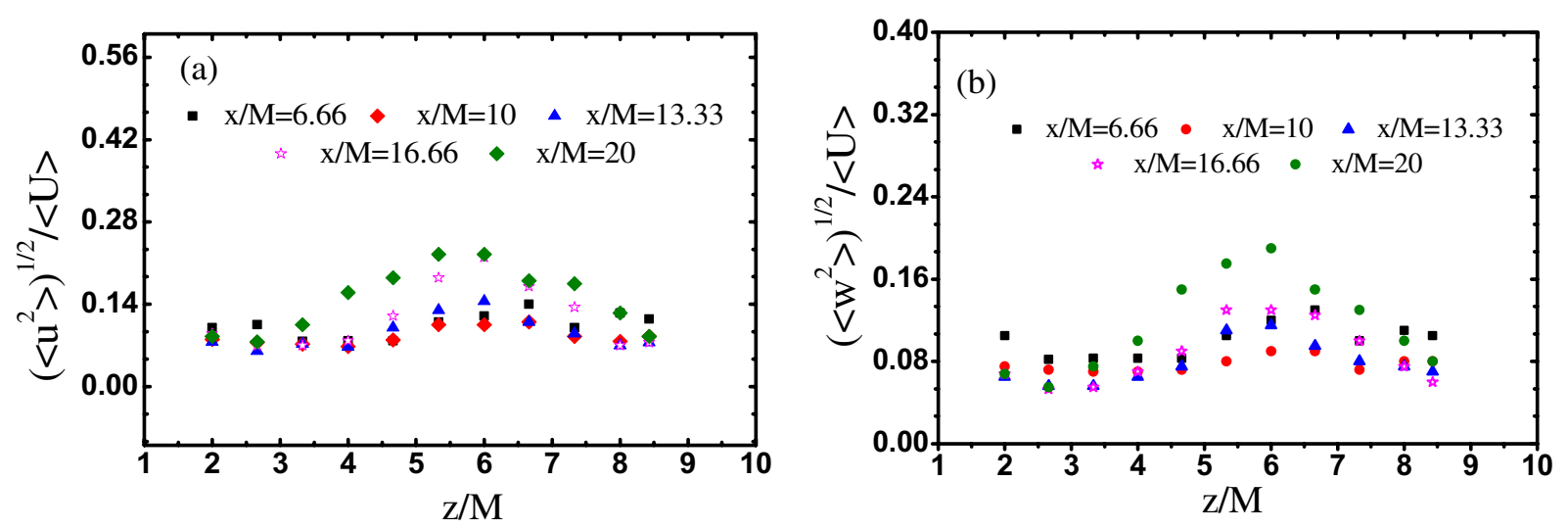

Fig. 6. Profiles of the turbulence rate, (a) longitudinal and (b) transversa.
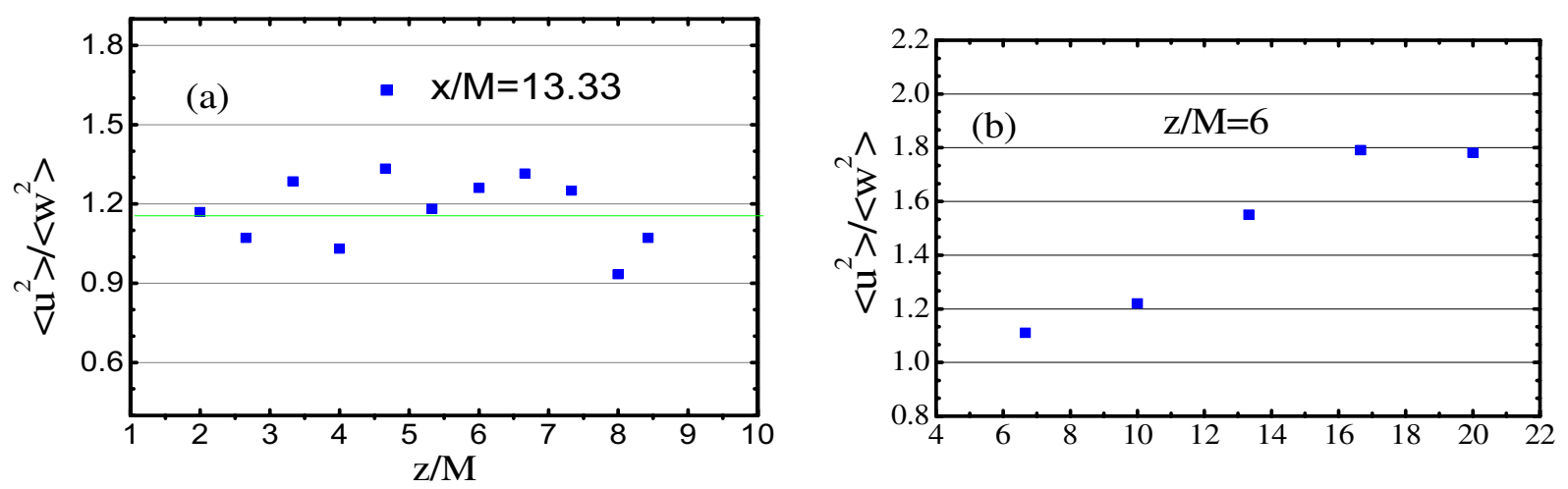

Fig. 7. Anisotropy factor at $x / M=13.33$, function of $z / M$, (b) function of $x / M$ at $z / M=6$.

$\left\langle u^{2}\right\rangle$, designated $D_{u u}$ and four deformation work terms designated $\Phi_{u u} ; D_{u u}$ includes the pressure-diffusion term, the transport by turbulent velocity fluctuations term and the transport by viscous stresses term. The deformation work terms $\Phi_{u u}$ including, turbulence production by the mean velocity gradients, buoyant production, pressionstrain and the dissipation. Except for the pressurecorrelations term, which is inferred from the budget, all of the other terms are derived from measurements.

As emphasized by Luminita [22], in any flow, even one characterized by inhomogeneities and anisotropies at large scales, there is a range of scales (even limited) that have locally homogeneous and isotropic properties. Therefore, the simplest and consequently most widely used technique to estimate $\epsilon_{u}$ is based on the assumption of local isotropy of the small scale structures together with the use of Taylor's hypothesis. Here, the mean dissipation rate $\epsilon_{u}$ is calculated from longitudinal one-dimensional spectra (1D) in the "inertial region" to the fluctuating velocity component $E_{u}\left(k_{1}\right)$, where $k_{1}=2 \pi f / U$ is the streamwise one dimensional wavenumber. The energy spectrum $E_{u}$ is related to $\left\langle u_{1}^{2}\right\rangle$ by $\left\langle u_{1}^{2}\right\rangle=\int_{0}^{\infty} E_{u}\left(k_{1}\right) d k_{1}$. Considering that a local isotropy $\epsilon_{u}$ can be evaluated via (1D) Fourier transforms of the velocity by $\epsilon_{u}=15 \nu \int_{0}^{\infty} k_{1}^{2} E_{u}\left(k_{1}\right) d k_{1}$ as shown by Antonia [23].
The value of pressure correlations deducted from the budget balance are higher than those found by Pavageau and Rey [24] who used Launder et al.'s model [25] to evaluate separately the pressure-diffusion and pressurestrain terms.

By examining the streamwise evolution of horizontally mean values of the statistical quantities involved in the balance equations of each section, the flow will be consider as if it was homogeneous horizontally.

The $\left\langle u^{2}\right\rangle$ budgets presented in Figure 8 reveal that the diffusion by turbulence (term 3 ) and the viscous diffusion (term 4) are low compared to the other balance terms but are not zero; they have a weak contribution to the energy. Production by the mean gradient increases downstream of the grid, achieving the same order of magnitude as production by buoyancy at the highest measurement section. This increase is mainly due to the high values of the mean velocity gradient in the upper flow region. Dissipation is the dominant term in first part of the flow, and it remains active in the higher region; nonetheless, it cannot balance the total turbulent energy production. Following this budget balance analysis, the evolutions of the streamwise mean velocity variance presented in Figure 5 is justified. 


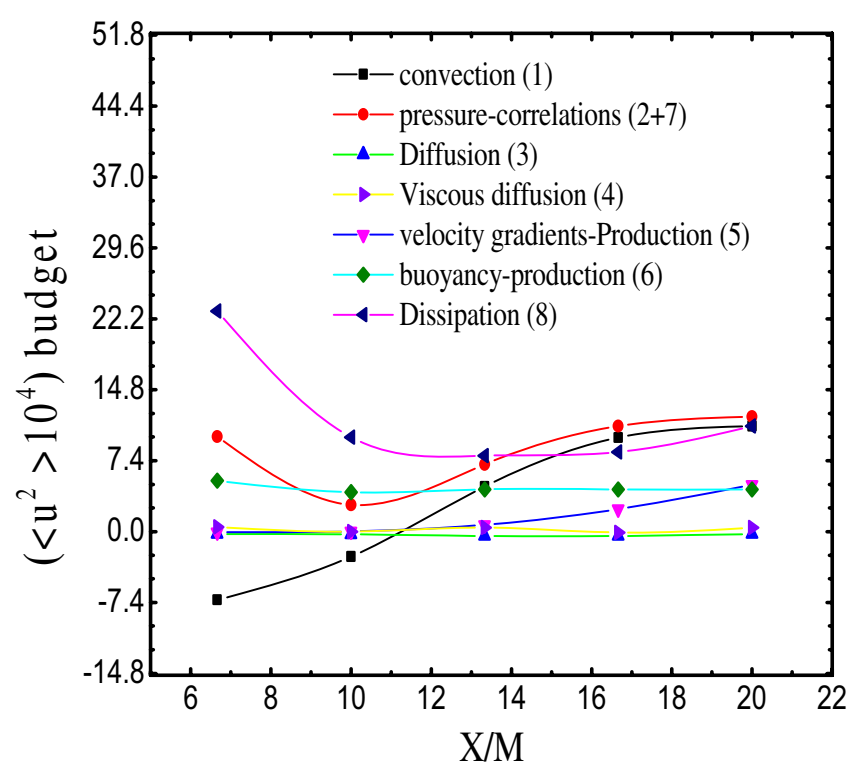

Fig. 8. Streamwise evolution of balance terms in the $\left\langle u^{2}\right\rangle$ energy equation.

\subsection{Distributions of fluctuations and mean thermal variances}

Figure 9 shows the transverse profiles of the thermal turbulence rate noting that the distribution is not homogeneous and that the temperature fluctuations exhibit a maxima at the centreline region. This is to be expected because temperature fluctuation production tends to increase with decreasing mean temperature gradients. In the following, $\left\langle\theta^{2}\right\rangle$ denotes the average of all of the values along the transverse section. Figure 10 shows the trend of temperature variance, which translates into a first decreasing phase of the thermal turbulent energy; this remains somewhat consistent with what is generally observed in forced convection (when the temperature is taken as a passive contaminate).

However, after $x / M=13.33$, a second phase occurs in which the decay stops and a slight increase is noticed downstream of the grid. The transport of a passive scalar contaminant in nearly isotropic turbulence was first theoretically investigated by Corrsin [3].

His initial inference that a transverse linear temperature gradient would remain linear as it was transported by the flow has been confirmed experimentally by Wiskind [26], Alexopoulos and Keffer [27], Venkataramani and Chevray [28] and Sirivat and Warhaft [7]. The first three papers report experiments with heated grids, while the last one reports experiments with two types of heating screens separate from the grid. Figure 11 replots the data from these studies. The values were obtained by averaging over the core of the flows; Venkataramani and Chevray [28] found, uniquely, that in their uniformgradient experiment, $\left\langle\theta^{2}\right\rangle$ first increased to a maximum value approximately 40 mesh lengths from the grid, after which it decreased; however, their experiment extends to only $x / M \approx 50$. Wiskind [26] and Alexopoulos

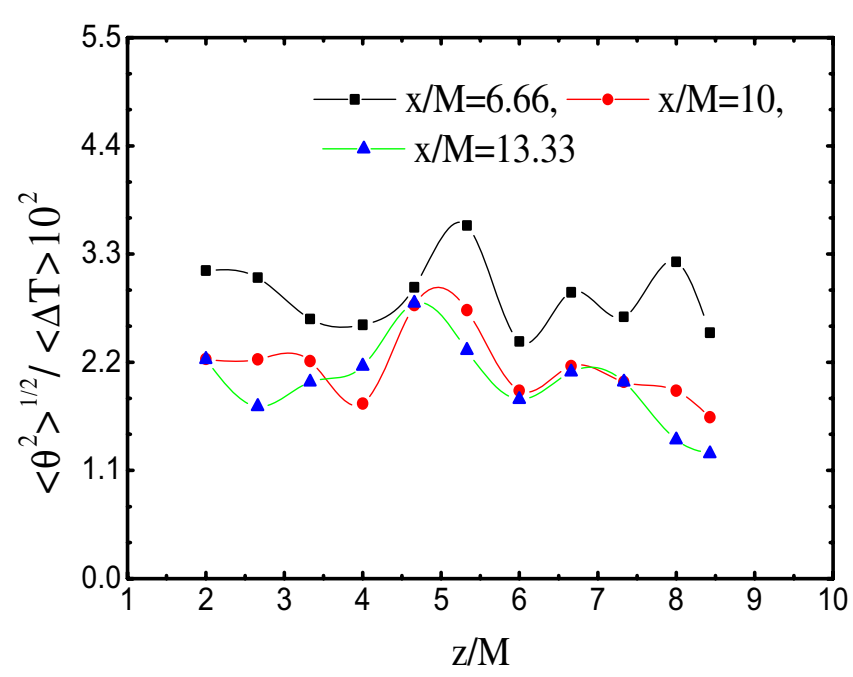

Fig. 9. Transverse profiles of the thermal turbulence rate $(\Delta T$ is the mean temperature rise maintained between upstream and downstream from the two grids).

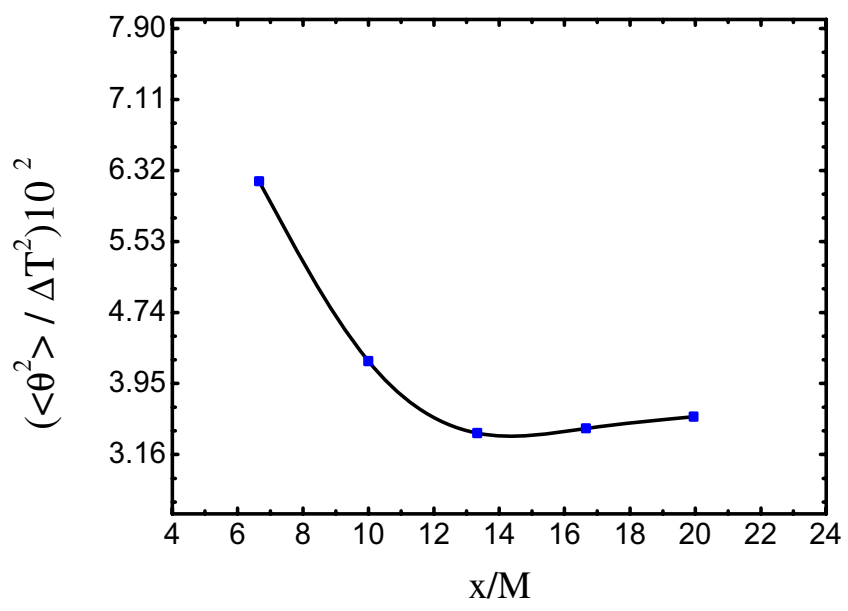

Fig. 10. The longitudinal thermal variances at $z / M=6$ neighbourhood of the centre-line of the channel.

and Keffer [27] showed an increase after approximately $x / M \approx 50$. Part of the reason for the large scatter in these data probably lies in the method used to generate the thermal turbulence. In fact, the method of producing the temperature gradient using differentially heated grid bars also produces an initial temperature variance, complicating the initial condition. According to the above, it is clear that after decay and beyond a specified distance downstream of the grid, an increase of turbulent thermal variance is observed. We can conclude that the experimental results in Figure 10 are in agreement with what was observed in the studies cited above when the temperature is taken as a passive contaminate. Although there is a difference between the quantitative values, the trends are preserved in all cases cited above 


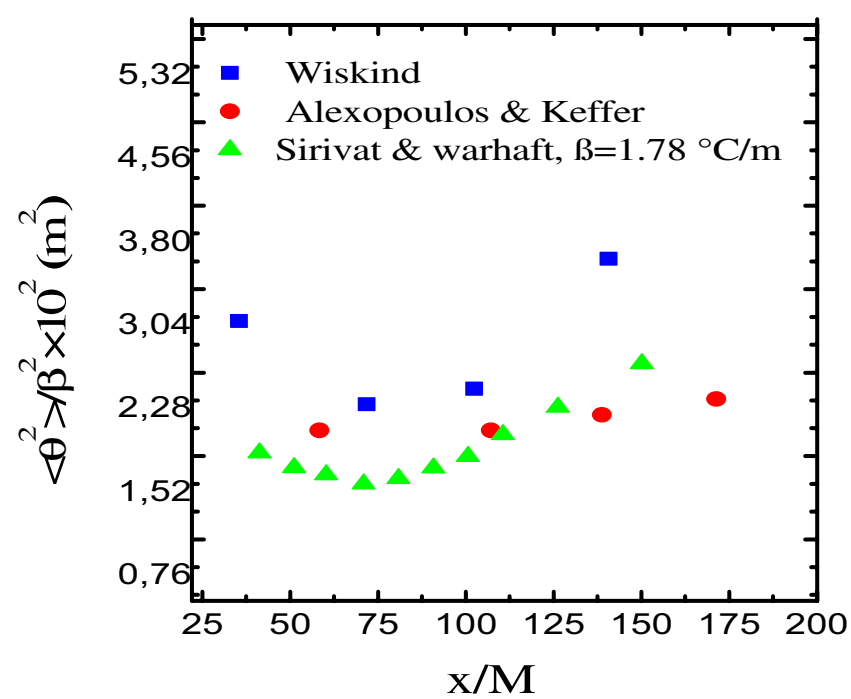

Fig. 11. Decaying $\left\langle\theta^{2}\right\rangle /\left\langle\beta^{2}\right\rangle$ in previous experiments with forced convection, extracted from Sirivat and Warhaft (1983). ( $\beta$ is a passive linear cross-stream temperature gradient).

\subsubsection{Transport equation of thermal variance budget}

In regards to the turbulent thermal energy, described in Figure 10, in way to express a local situation that could be obtained if the flow was homogeneous in each horizontal plan; we have assumed that each value of $\left\langle\theta^{2}\right\rangle$ is equivalent to the integral of the values over a transversal measurement line.

To understand why the turbulent thermal energy does not continue to decay far downstream of the grid, it is instructive to examine the balance of transport equation of $\left\langle\theta^{2}\right\rangle$.

The dimensional form of the transport equation of the temperature variance $\left\langle\theta^{2}\right\rangle$ is given by

$$
\langle U\rangle \frac{\partial\left\langle\theta^{2}\right\rangle}{\partial x}=P_{\theta}+d_{\theta}-2 \epsilon_{\theta}
$$

The term on the left-hand side is the mean flow convection representing the production of thermal variance by turbulent stirring of the flow. On the right-hand side, the symbols $P_{\theta}, D_{\theta}$ and $\varepsilon_{\theta}$ note the production rate, the diffusion terms composed of the transport turbulent diffusion and transport by molecular diffusion and the dissipation rate of the temperature fluctuations, respectively. The dimensional form of the transport equation of the temperature variance $\left\langle\theta^{2}\right\rangle$ is given by

$$
\begin{array}{r}
\langle U\rangle \frac{\partial\left\langle\theta^{2}\right\rangle}{\partial x}=\underbrace{-2\langle u \theta\rangle \frac{\partial\langle T\rangle}{\partial x}}_{P_{\theta}}+\underbrace{\frac{\partial}{\partial x}\left[\alpha \frac{\partial\left\langle\theta^{2}\right\rangle}{\partial x}-\left\langle\theta^{2} u\right\rangle\right]}_{D_{\theta}} \\
-2 \underbrace{2\left\langle\left(\frac{\partial \theta}{\partial x}\right)\left(\frac{\partial \theta}{\partial x}\right)\right\rangle}_{\epsilon_{\theta}}
\end{array}
$$

where $\alpha$ is the molecular diffusivity and $\gamma$ is the thermal diffusivity. $P_{\theta}$ is evaluated as the product of the temperature flux and the mean gradient.

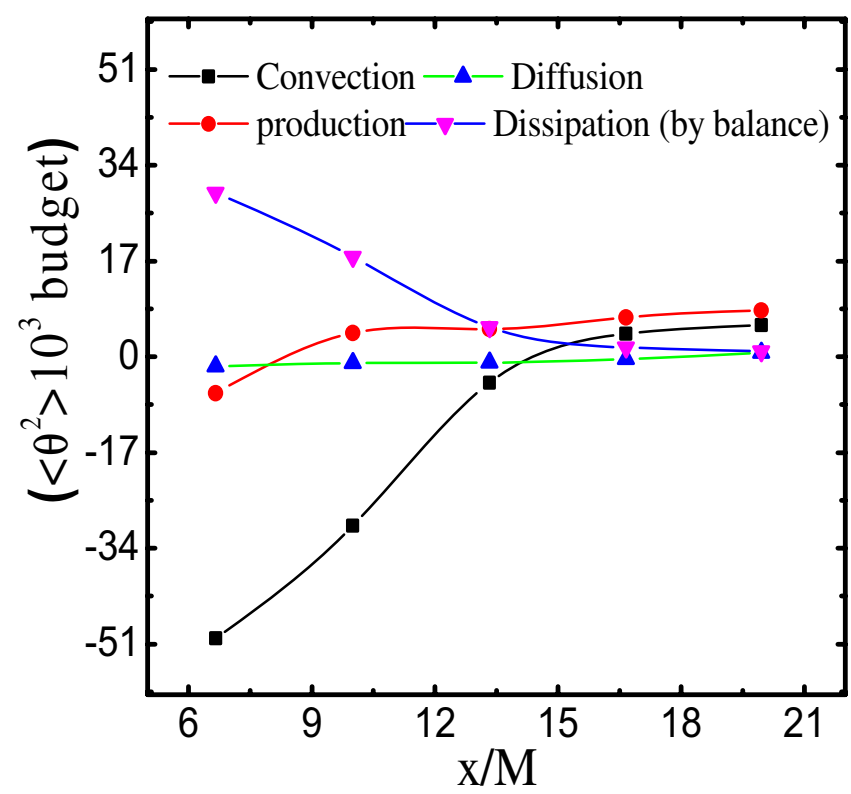

Fig. 12. Temperature variance budgets.

The transport turbulent diffusion term is measured, the molecular-transport term is estimated to be approximately $10^{-7}$ with a weak contribution to the balance. Instead of solving an additional equation for the dissipation rate of the temperature variance (which is a challenging task), $\epsilon_{\theta}$ is inferred from the balance. All terms of the transport equation are represented in Figure 12 at five upstream stations of the grid. With reference to the previous equation and the data of Figure 10, we can conclude that the temperature variance first decreases under the dissipation effect, after passing through a minimum, and increases as the thermal production begins to dominate dissipation. Although the turbulence found in natural and technological flows is ordinarily far from homogeneous and isotropic, many features of diverse turbulent flows, especially the spatially local features, seem to be moderately universal. The decay in power law of variance $\left\langle\theta^{2}\right\rangle$ similar to variance $\left\langle u^{2}\right\rangle$ is now accepted. The use of power laws in the description of grid turbulence is a well-established practice (e.g., Warhaft and Lumley [6]; Sreenivasan et al. [29]; Mohamed and LaRue [21]; and Antonia et al. [12].

In the present flow, although the turbulence is neither homogeneous nor isotropic, in the following, we propose to describe the first part of the flow using a power law. Figure 13 shows the temperature fluctuation intensity as a function of $x / M$ in the vicinity of the central channel axis (at $z / M=6$ ). For convenience, the second point of the curve is determined by extrapolation. In the first part of the flow $x / M \leq 13.33$, the temperature variance decreases, and the relation on a log-log plot can be approximated by a straight line according to the following power law

$$
\left\langle\theta^{2}\right\rangle /\left\langle\Delta T^{2}\right\rangle=B(x / M)^{-m}
$$




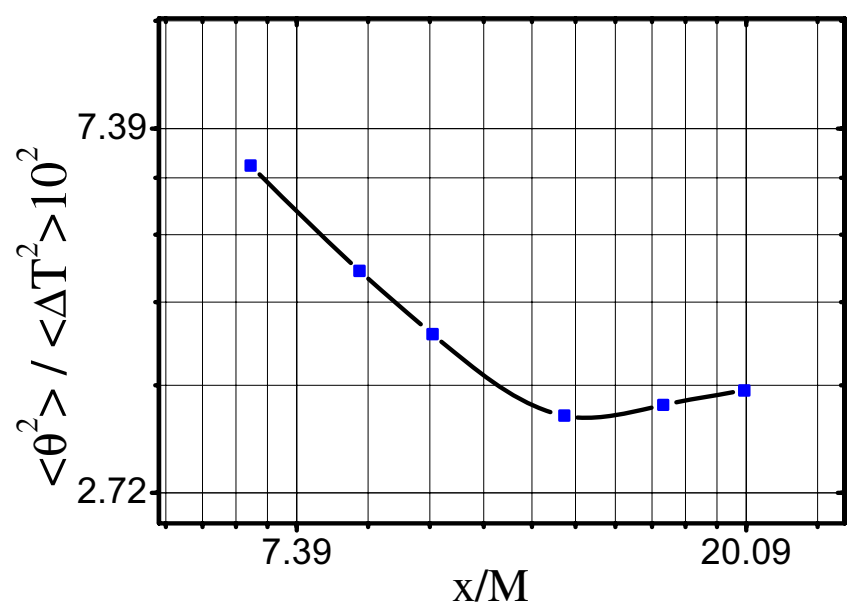

Fig. 13. The longitudinal thermal variances in the vicinity of the centre-line $(z / M=6)$.

where $B$ is the decay coefficient, $m$ is the exponent decay and $x$ is the coordinate, which is positive in the downstream direction and is usually measured from a virtual origin. By applying the least-squares linear fitting equation, we note that the decay coefficient is $B=0.417$ and the exponent decay is $m=-0.873$. The form of the decay power-law expression in this area is written as

$$
\left\langle\theta^{2}\right\rangle /\left\langle\Delta T^{2}\right\rangle=0.417(x / M)^{-0.873}
$$

The usual similarity established for all streamwise flow in forced turbulent grid is not found in our configuration.

\section{Conclusion}

The present investigation on grid-generated turbulence purely driven by thermal convection, showed that turbulence remains spatially inhomogeneous even far downstream of the grid. In fact, far downstream of the grid, the inhomogeneity of the mean velocity and of the turbulence fluctuations persists. Of course, this affects the rate of decay of turbulent energies.

An increase in the variance of the velocity fluctuations is observed in contrast to what is usually known in homogeneous and isotropic forced grid turbulence. Moreover, the streamwise decay of the temperature variance typical of the forced convection grid turbulence is not observed. This difference in the evolution of turbulent energies is probably due to the fact that when mean velocity gradients far downstream of the grid increase, the turbulence production rapidly become active.

In order to elucidate the possible reasons for this contrast, we analysed the results of our experiments by evaluating the terms of the transport equations budget of the kinetic and thermal energies. We notice that, the dissipation dominant term in the first part of the flow is still active in the higher region of the flow. Nonetheless, this remaining viscous dissipation term is insufficient to balance the total turbulent energy production.
For our experimental set up, the channel height was not large enough to allow the flow to reach the fully developed conditions. So, the transient region probably extends much farther than for classical wind-tunnel grid generated turbulence.

To obtain the usual decay of turbulence in the present flow, a higher channel would be necessary and an adjustable channel walls would have been required. This would ensure a constant centreline mean velocity when the boundary layer develops and would best approximate an isotropic flow.

Acknowledgements. The experimental work was conducted in the Fluid Mechanics Laboratory (Ecole Centrale de Nantes, France, UMR 6598, CNRS). We gratefully acknowledge Pr. Claude Rey for his support.

\section{References}

[1] G.I. Taylor, Statistical theory of turbulence, 4-diffusion in a turbulent air stream, Proc. R. Soc. Lond. Ser. A Math. Phys. Sci. 51 (1935) 465-478

[2] G.K. Batchelor, The theory of homogeneous turbulence. Cambridge University Press, Cambridge, 1953

[3] S. Corrsin, The decay of isotropic temperature fluctuations in an isotropic turbulence, J. Aeronaut. Sci. 18 (1951) 417-423

[4] R.R. Mills, A.L. Kistler, V. O'Brien, S. Corrsin, Turbulence and temperature fluctuations behind a heated grid. Tech. Note 4288, National Advisory Committee for Aeronautics, 1958

[5] R.R. Mills, S. Corrsin, Effects of contraction on turbulence and temperature fluctuations generated by a warm grid. N.A.S.A. Memo. no. 5-5-59W, 1959

[6] Z. Warhaft, J.L. Lumley, An experimental study of the decay of temperature fluctuations in grid-generated turbulence, J. Fluid Mech. 88 (1978) 659-684

[7] A. Sirivat, Z. Warhaft, The effect of a passive crossstream temperature gradient on the evolution of temperature variance and heat flux in grid turbulence, J. Fluid Mech. 128 (1983) 323-346

[8] W.K. George, The decay of homogeneous isotropic turbulence, Phys. Fluids 4 (1992) 1492-1509

[9] M.M. Gibson, T. Dakos, Production of temperature fluctuations in grid turbulence: Wiskind's experiment revisited, Experiments in Fluids 16 (1993) 146-154

[10] P. Lavoie, L. Djenidi, R.A. Antonia, Effects of initial conditions in decaying turbulence generated by passive grids, J. Fluid Mech. 585 (2007) 395-420

[11] R.A. Antonia, P. Lavoie, L. Djenidi, A. Benaissa, Effect of a small axisymmetric contraction on grid turbulence, Exp. Fluids 49 (2010) 3-10

[12] S.K. Lee, A. Benaissa, L. Djenidi, P. Lavoie, R.A. Antonia, Decay of passive- scalar fluctuations in slightly stretched grid turbulence, Exp. Fluids 53 (2012) 909-923

[13] G. Comte-Bellot, S. Corrsin, The use of a contraction to improve the isotropy of grid generated turbulence, J. Fluid Mech. 25 (1966) 657-682

[14] S. Viswanathan, S.B. Pope, Turbulent dispersion from line sources in grid turbulence, Phys. Fluids 20 (2008) 101514 
[15] T.F. Ayinde, S.A.M. Said, M.A. Habib, Experimental investigation of turbulent natural convection flow in a channel, Heat Mass Transf. 42 (2006) 169-177

[16] M.S. Uberoi, S. Wallis, Spectra of Grid Turbulence, Phys. Fluids 12 (1969) 1355-1358

[17] Ö. Ertunç, N. Özyilmaz, H. Lienhart, F. Durst, K. Beronov, Homogeneity of turbulence generated by staticgrid structures, J. Fluid Mech. 654 (2010) 473-500

[18] S. Corrsin, Turbulence: Experimental Methods, Springer, vol. 8., 1963

[19] L. Djenidi, T. Tardu, On the anisotropy of a lowReynolds-number grid generate turbulence, J. Fluid Mech. 702 (2012) 332-353

[20] M.S. Mohamed, J.C. LaRue, The decay power law in gridgenerated turbulence, J. Fluid Mech. 219 (1990) 195-214

[21] T.J. Craft, N.Z. Ince, B.E. Launder, Recent developments in second-moment closure for buoyancy-affected flows, Dynamics of Atmospheres and Oceans 23 (1996) 99-114

[22] L. Danaila, A. Fabien, T. Zhou, R.A. Antonia, Transport equation for the homogeneous mean energy dissipation rate in decaying grid turbulence, Mécanique Industries 4 (2003) 415-420
[23] R.A. Antonia, On estimating mean and instantan- neous turbulent energy dissipation rates with hot wires, J. Fluid Mech. 505 (2003) 123-151

[24] M. Pavageau, C. Rey, Observation of volume variation effects in turbulent free convection, Int. J. Heat Mass Transf. 45 (2002) 181-192

[25] B.E. Launder, G.J. Reece, W. Rodi, Progress in the development of Reynolds-stress turbulent closure, J. Fluid Mech. 68 (1975) 537-566

[26] H.K. Wiskind, A uniform gradient turbulent trans-port transport experiment, J. Geophys. Res. 67 (1962) 30333048

[27] C.C. Alexopoulcos, J.F. Keffer, Turbulent wake in a passively stratified field, Phys. Fluids 14 (1971) 216-224

[28] K.S. Venkataramakn, R. Chevray, Statistical features of heat transfer in grid generated turbulence - constantgradient case, J. Fluid Mech. 86 (1978) 513-543

[29] K.R. Sreenivasan, S. Tavoularis, R. Henry, S. Corrsin, Temperature fluctuations and scales in grid-generated turbulence, J. Fluid Mech. 100 (1980) 597-621 